\title{
Tunability and Robustness of Dirac Points of Photonic Nanostructures
}

\author{
Hanying Deng, Xianfeng Chen, Boris A. Malomed, Nicolae C. Panoiu and Fangwei Ye
}

(Invited Paper)

\begin{abstract}
We study the tunability and robustness of photonic Dirac points (DPs) in plasmonic nanostructures. The tunability of the DP is demonstrated in graphene-based photonic superlattices by adjusting the graphene permittivity via the optical Kerr effect or electrical doping. The robustness of DPs is demonstrated in plasmonic lattices by showing that even very high levels of disorder are unable to localize the modes located near the Dirac point. The robustness of the DP also manifests itself in the fact that the inversely-proportional dependence of the transmission on the lattice length near the Dirac point remains unchanged under strong disorder.
\end{abstract}

Index Terms-Graphene, photonic superlattices, Dirac points, structural disorder, transmission.

\section{INTRODUCTION}

$\mathbf{P}$ ERIODIC photonic systems, through a proper design of their photonic band structure, provide unprecedented opportunities for manipulating the interaction between light and matter. In these systems, various exotic photonic band structures can be engineered, featuring frequency gaps, spectral domains with strong anisotropy, negative refraction, and Dirac points (DPs). In the case of DPs, whose properties are the subject of the present paper, an upper and lower photonic bands are designed in such a way that they intersect at a single point, the frequency dispersion of the optical modes located in the vicinity of this point being linear. Interestingly, it has been recently demonstrated that the band structure of surfaceplasmon polaritons (SPPs), which occur in one-dimensional (1D) layered metallic-dielectric nanostructures, possess DPs provided that the spatial average of the permittivity of the lattice is zero [1]-[3]. In condensed-matter physics, electronic counterparts of photonic DPs are at the origin of many remarkable properties of recently discovered materials, such as graphene and topological insulators [4]-[6]. Therefore, the ability to create, eliminate, and, more generally, manipulate DPs of photonic structures could have many important implications,

The work of Hanying Deng and Fangwei Ye was supported by Innovation Program of Shanghai Municipal Education Commission (Grant No. 13ZZ022) and the National Natural Science Foundation of China (Grants No. 11104181 and No. 61475101). The work of Nicolae C. Panoiu was supported by a European Research Council / ERC Grant Agreement no. ERC-2014-CoG648328. (Corresponding author: Fangwei Ye.).

Hanying Deng, Fangwei Ye and Xianfeng Chen are with the Department of Physics and Astronomy, Shanghai Jiao Tong University, Shanghai 200240, China (e-mail: dhy0805@sjtu.edu.cn; fangweiye@sjtu.edu.cn; xfchen@sjtu.edu.cn).

Nicolae C. Panoiu is with the Department of Electronic and Electrical Engineering, University College London, Torrington Place, London WC1E7JE, UK (e-mail: n.panoiu@ucl.ac.uk).

Boris A. Malomed is with the Department of Physical Electronics, School of Electrical Engineering, Faculty of Engineering, Tel Aviv University, Tel Aviv 69978, Israel (e-mail: malomed@post.tau.ac.il). both at the fundamental level and to photonic devices with new or improved functionalities. The photonic DPs provide a convenient way to explore and understand DP-related physics in the general form, as photonic structures offer a versatile, easy to use platform for the experimental implementation of such physical systems [7]. However, a major problem impeding the implementation of this approach is that both photonic and plasmonic crystals usually lack tunability of their performance.

A recently introduced photonic platform that contains all the ingredients needed to achieve ultra-fast broad optical tunability at the nanoscale is graphene [8], [9].Compared to the SPPs in metals, graphene plasmons are localized in much smaller regions, provide a much larger enhancement of optical near-fields, and can propagate significantly longer distances [10]. Equally important, the conductivity of graphene and, implicitly, its local electrical permittivity can be substantially varied by means of chemical doping or gate voltage [11], [12]. The latter method makes it also possible to implement ultrafast variation of graphene permittivity. For example, changing the Fermi level by a mere $1 \mathrm{eV}$ causes the relative variation of the real part of the permittivity, at $10 \mu \mathrm{m}$, by $250 \%$. Depositing graphene onto the surface of specific material$\mathrm{s}$, which may be both dielectrics and metals, a variety of graphene-based layered nanostructures have been designed, and their implementations in functional photonic devices have been demonstrated [13]-[15]. The most salient feature of these superlattices is that, by incorporating graphene into the periodic structure, the spectral band structure and, thus, their optical response can be dynamically tuned, using the above-mentioned method of electric gating. This key property, in conjunction with relatively low loss and extremely tight confinement of the graphene plasmons, opens up promising applications of tunable graphene photonic superlattices (GPSs) at mid-IR and at $\mathrm{THz}$ wavelengths. In Section II, we present an example of such an application, demonstrating tunability of DPs in graphene-based superlattices.

Generally speaking, photonic DPs can be divided into two broad classes according to how they appear. One class encompasses structural DPs, [16] whose existence is related to specific topological and structural properties of the underlying photonic lattice. In particular, such DPs, occurring in honeycomb lattices regardless of their parameters, may be viewed as the photonic analog of the electronic DPs of graphene and other two-dimensional (2D) materials. The other class of photonic DPs includes the so-called accidentaldegeneracy-induced DPs (ADIDPs) [17]. They can appear in simple lattices, such as square ones, when the lattice 
parameters (such as the spacing and refractive-index contrast) are finely tuned in such a way that the effective permittivity and permeability of the structure vanish at specific frequencies. The above-mentioned DPs occurring in 1D plasmonic lattices with vanishing average permittivity belong to the ADIDPs class, too.

In reality, however, fabrication processes inevitably introduce a certain level of structural disorder upon producing periodic structures, and thus it is important to analyze the survival of the DPs under the structural disorder, thus securing the experimental observations of the DP-related physics. Effects of disorder on the structural DPs have been investigated in the particular case of optical honeycomb waveguide lattices, the main finding being that the DP-associated chiral symmetry is preserved or broken, depending on the nature of the disorder [18]. The influence of the disorder on ADIDPs is considered in Section III. We find that the DPs of this type are very robust, in the sense that even very high disorder levels are unable to induced the (Anderson) localization of modes close to the DP.

Section IV addresses the transmissivity of finite-size plasmonic lattices that contain DPs in their spectral band structure (in the perfectly-periodic limit). It has been previously reported that the transmissivity close to the DP is characterized by scaling $\sim 1 / L$ with respect to length $L$ of the lattice [19], [20]. Such $1 / L$ scaling of the transmissivity is called "pseudo-diffusive", being reminiscent of the diffusion through a disordered medium, but we stress that here it occurs in the regular lattice in the absence of any disorder. Next, in Section IV we address the transmissivity of the disordered DP-bearing plasmonic lattice. We find that the $1 / L$ scaling remains unchanged near the DPs, providing another proof of the their robustness against the structural disorder.

\section{TUNABILITY OF THE DIRAC POINT}

The GPS concept, that was put forward in Ref. [21], not only enables manipulations of the light-matter interactions at the deep-subwavelength scale, but also provides unprecedented tunability of their functionalities and performance. The advantage of including graphene sheets into the unit cell of metallo-dielectric superlattices is that one can then readily design GPSs that posses electrically and optically tunable DPs. To add specificity to these ideas, we consider, as an example of a GPS, the 1D periodic structure displayed in Fig. 1(a). It has a three-layer unit cell, graphene-metal-dielectric, the metallic and dielectric layers chosen here being silver and silicon, respectively. The dispersion relation of the structure is easily found by using the transfer-matrix method [22], and is given by (see also Appendix A):

$$
\begin{aligned}
& \cos \left(k_{x} \Lambda\right)=\sigma\left(1-\frac{\varrho_{g d}^{2} \varrho_{d g}^{2}}{2 \varrho_{g d} \varrho_{d g}} \tan \kappa_{g} \tan \kappa_{d}-\right. \\
& \left.\frac{\varrho_{d m}^{2}+\varrho_{m d}^{2}}{2 \varrho_{d m} \varrho_{m d}} \tan \kappa_{d} \tan \kappa_{m}-\frac{\varrho_{m g}^{2}+\varrho_{g m}^{2}}{2 \varrho_{m g} \varrho_{g m}} \tan \kappa_{m} \tan \kappa_{g}\right),
\end{aligned}
$$

where $k_{x}$ is the Bloch wave vector, $k_{z}$ the propagation wave vector, $t_{g}, t_{d}$, and $t_{m}$ are thicknesses of the layers, $\Lambda=t_{g}+$
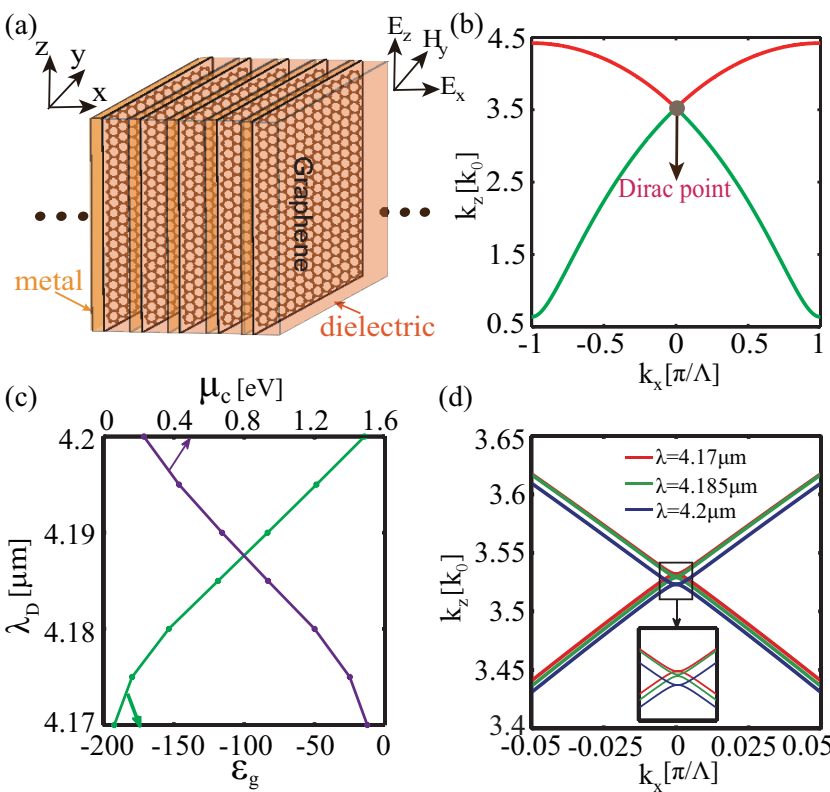

(d)

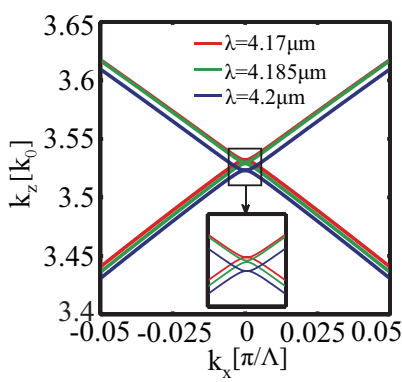

Fig. 1. (a) A schematic of the photonic superlattice: the unit cell is a graphene-metal-dielectric tri-layer configuration stacked along the $x$ direction. The incident TM-polarized beam propagates along the $z$-axis, its nonvanishing field components being $E_{x}, E_{z}$, and $H_{y}$. (b) The photonic band structure calculated in the case of the zero-averaged-permittivity, featuring the 1D Dirac point. The structural parameters are $\varepsilon_{g}=-14.48, \varepsilon_{d}=12.25$, $\varepsilon_{m}=-924.05, t_{g}=0.5 \mathrm{~nm}, t_{d}=604 \mathrm{~nm}, t_{m}=8 \mathrm{~nm}$, and $\lambda_{D}=4.2 \mu \mathrm{m}$. (c) The dependence of the wavelength of the Dirac point on the graphene's chemical potential and permittivity. (d) The photonic band structure calculated for different wavelengths.

$t_{d}+t_{m}$ is the period of the supercell, $\sigma \equiv \cos \kappa_{g} \cos \kappa_{d} \cos \kappa_{m}$, $\kappa_{j} \equiv t_{j} \sqrt{(\omega / c)^{2} \varepsilon_{j} \mu_{j}-k_{z}^{2}}$, and $\varrho_{i j} \equiv \kappa_{i} \varepsilon_{j} t_{j}$, with $j=$ $g, d, m$ standing for graphene, dielectric (silicon), and metal (silver), respectively. By fixing the frequency $\omega$ in Eq. (1), the dependence $k_{z}=k_{z}\left(k_{x}\right)$ defines the spatial dispersion relation for the particular frequency.

To produce a DP, the band structure of such GPSs should, first, possess two transmission bands. Further, for a two-band configuration to occur, the thickness of the dielectric and metallic layers should be larger than certain critical values. For example, for $t_{m}=8 \mathrm{~nm}$, in order to support the twoband structure, the minimum thickness of dielectric layers is $t_{d}=592 \mathrm{~nm}$. Then, similar to other plasmonic structures, the two bands intersect at the center of the Brillouin zone, $k_{x}=0$, giving rise to the photonic DP [see Fig. 1(b)], provided that the real part of the average permittivity of the superlattice vanishes [1]-[3], that is,

$$
\mathfrak{R e}(\bar{\varepsilon})=\frac{1}{\Lambda} \mathfrak{R e}\left(\varepsilon_{g} t_{g}+\varepsilon_{d} t_{d}+\varepsilon_{m} t_{m}\right)=0 .
$$

We now demonstrate that the graphene component of this photonic superlattice allows one to tune the wavelength of the photonic DPs, by merely varying the chemical potential of graphene, $\mu_{\mathrm{c}}$. More specifically, changing $\mu_{\mathrm{c}}$ by means of external tools (gate voltage, chemical doping, etc.), one alters the graphene's permittivity, making it possible to tune the wavelength, $\lambda_{D}$, at which condition $\mathfrak{R e}\left[\bar{\varepsilon}\left(\lambda_{D}\right)\right]=0$ holds. These ideas are illustrated in Fig. 1(c), where we plot the dependence of the DP wavelength on $\mu_{\mathrm{c}}$. Note that a spectral 

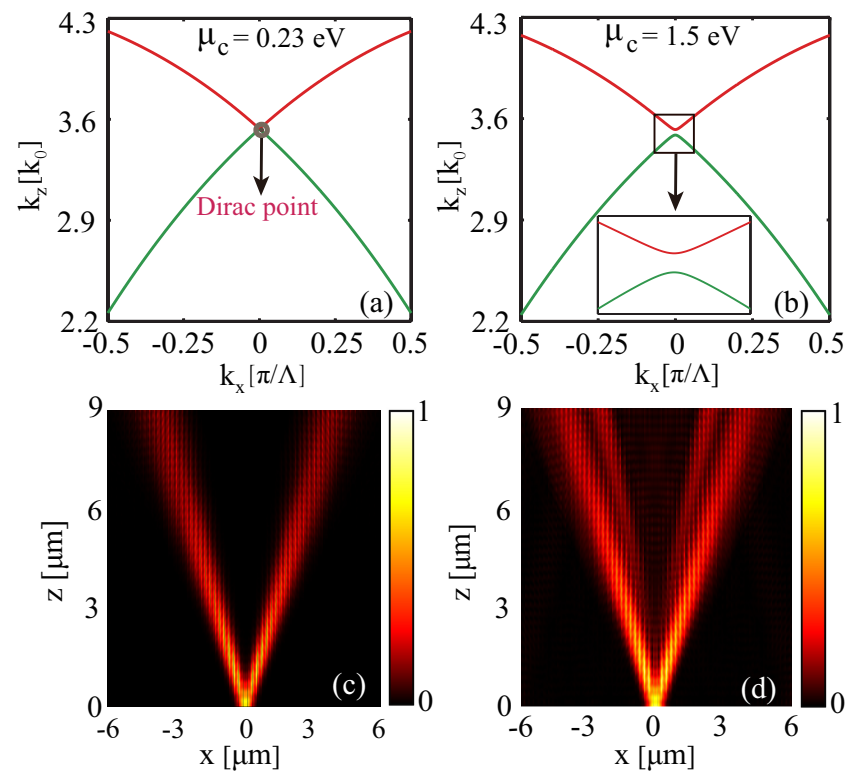

Fig. 2. (a,b) Formation of a bandgap in place of the Dirac point with $\lambda_{D}=4.2 \mu \mathrm{m}$ by varying the graphene's Fermi level. (c,d) Splitting of the incident TM-polarized Gaussian beam with profile $E_{x}(x)=\exp \left(-x^{2} / w^{2}\right)$, that has $w=2 \lambda_{D}$. In (a) and (c), permittivities of the layers are the same as in Fig. 1. In (b) and (d), $\varepsilon_{g}=-195.02$. In all cases, thicknesses of the layers are the same as in Fig. 1.

shift of more than $30 \mathrm{~nm}$ can be achieved changing $\mu_{\mathrm{c}}$ by $1.5 \mathrm{eV}$. The variation of the corresponding band structure is presented in Fig. 1(d).

The tunability of the band-structure of the GPS provides an effective way to control the excitation and propagation of optical beams. As Figs. 2(a) and 2(b) show, when $\mu_{\mathrm{c}}$ varies from $0.23 \mathrm{eV}$ to $1.5 \mathrm{eV}$, the band structure transforms from a gapless one, which possesses a DP, to a gapped structure, this effect being accompanied by a dramatic change in the beam-propagation dynamics. At the DP, a normally incident Gaussian beam splits into two secondary ones only, as the upper- and lower-band excitations are not resolved, due to the fact that the two bands have identical slopes near the DP [see Fig. 2(c)]. By contrast, when a gap opens the output pattern exhibits four split beams, due to the difference in the slopes of the upper and lower bands near $k_{x}=0$, as per Fig. 2(d).

In addition to the electric means, the wavelength at the DPs can be tuned by employing the intrinsic optical nonlinearity of graphene, as well as that of the dielectric and metallic layers. Such nonlinearity-induced tunability of the DP is possible in the presently considered superlattices because, as shown above, the DPs appear when the real part of the average permittivity is zero. Accordingly, nonlinear optical effects, that change the local refractive index, can be used to tune the permittivity to or off the zero-average value, thus providing alloptical means to control the formation of the DPs. The optical nonlinearity of graphene is expected to be particularly strong, due to its unusually large third-order nonlinear susceptibility [30], as well as tightly localized optical field supported by graphene sheets.

To illustrate this possibility, Fig. 3 shows the creation/destruction of a DP under the action of the optical Kerr
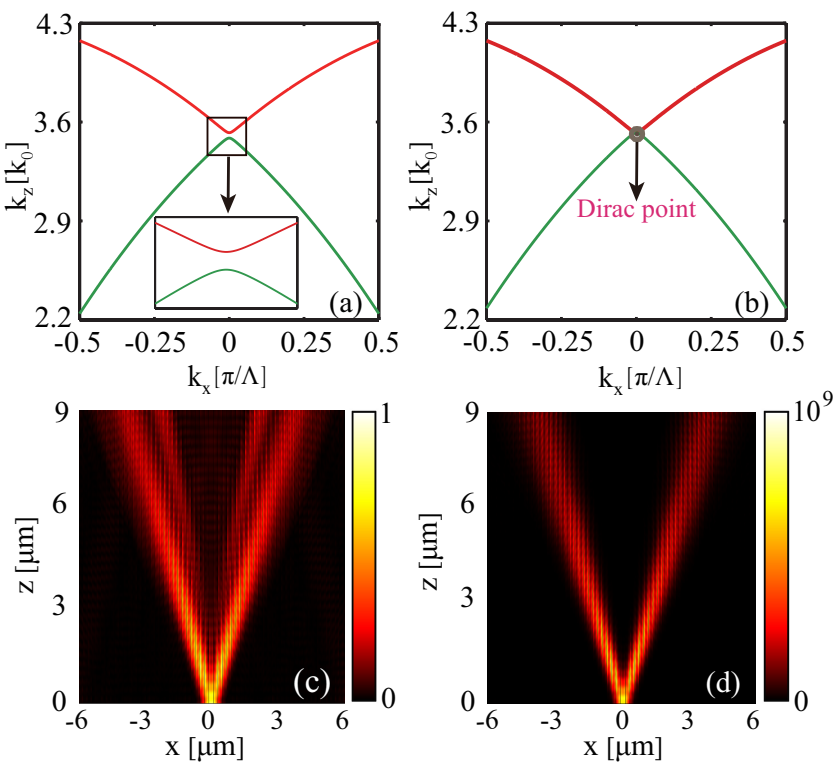

Fig. 3. (a) The linear band structure, calculated for the same parameters as in Fig. 1, except for $\varepsilon_{g}=-195.02(\bar{\varepsilon}=-0.15)$. (b) The nonlinear band structure, where the Bloch mode of the upper band at $k_{x}=0$ is used to induce the nonlinear (Kerr) change of permittivities, $\delta \varepsilon_{d}=0.07$ and $\delta \varepsilon_{g}=$ 0.46. (c,d) Splitting of the incident TM-polarized Gaussian beam, $E_{x}(x)=$ $A \exp \left(-x^{2} / w^{2}\right)$, with $w=2 \Lambda$ and $A=1 \mathrm{~V} / \mathrm{m}$ in (c) or $A=4.13 \times$ $10^{7} \mathrm{~V} / \mathrm{m}$ in (d). The Kerr coefficients are $n_{2, d}=1.86 \times 10^{-20} \mathrm{~m}^{2} / \mathrm{V}^{2}$ (for silicon) and $n_{2, g}=4.09 \times 10^{-17} \mathrm{~m}^{2} / \mathrm{V}^{2}$.

effect, acting in graphene and the adjacent dielectric (silicon) layer. At low optical powers, starting with a superlattice for which $\bar{\varepsilon}(\lambda)<0$, the two transmission bands are separated by a gap, as shown in Fig. 3(a). When the input power increases, both graphene and dielectric are affected by the Kerr nonlinearity, their permittivities varying by $\delta \varepsilon_{g}>0$ and $\delta \varepsilon_{d}>0$, respectively. As a result, the average permittivity, $\bar{\varepsilon}$, increases and the corresponding band structure is modified as well. At some value of the optical power, $\bar{\varepsilon}$ vanishes, so that, as shown in Fig. 3(b), the photonic gap closes and the DP emerges. For the particular case shown in Fig. $3, \delta \varepsilon_{d}=0.07$ and $\delta \varepsilon_{g}=0.46$. The propagation of a TM-polarized Gaussian beam, both in the linear and nonlinear regimes, is displayed in Figs. 3(c) and 3(d), respectively. One can see that, as the power of the input beam increases, the dynamics changes from typical discrete diffraction to $1 \mathrm{D}$ conical diffraction, which is a manifestation of the formation of the DP in the latter case.

\section{RoBUStNesS OF DiRAC POINTS AGAINST STRUCTURAL DISORDER}

Similar to zero- $\bar{n}$ band gaps, which are formed when the spatial average of the refractive index of a Bragg grating vanishes [23]-[29], ADIDPs in our photonic structure are particularly robust against disorder added to the system. This behavior is expected because structural disorder weakly affects the spatial average of the permittivity, hence its vanishing, which determines the existence of DPs, persists in the presence of disorder as well.

To evaluate the effect of structural disorder on the ADIDP$\mathrm{s}$, we here perform a comparative study of the Anderson localization of light waves in a disordered 1D plasmonic 
crystal that, in the regular (disorder-free) form, possesses either a DP or a Bragg gap (BG) in the spectrum of the wave vectors. For this purpose, we consider a binary periodic nanostructure composed of alternating metallic and dielectric layers(the results for section III and IV both correspond to the metallic-dielectric binary periodic structure, namely, when the graphene component in the structure shown in Fig. 1(a) is absent). As mentioned above, the 1D DP appears at the center of the Brillouin zone, $k_{x}=0$, provided that the real part of the spatially averaged permittivity vanishes, i.e., $\mathfrak{R e}(\bar{\varepsilon})=\left(t_{d}+t_{m}\right)^{-1} \mathfrak{R e}\left\{\varepsilon_{d} t_{d}+\varepsilon_{m} t_{m}\right\}=0$. To be specific, we assume that the metallic and dielectric layers are made (as above) of silver and silicon, respectively. The silicon permittivity is given by the above-mentioned value, $\varepsilon_{d}=12.25$, and the permittivity of silver is $\varepsilon_{m}=-125.39+2.84 i$ at the standard telecomm wavelength, $\lambda=1550 \mathrm{~nm}$. We further fix the thickness of the silicon layer as $t_{d}=256 \mathrm{~nm}$. With these parameters, it is readily found that, for $t_{m}=25 \mathrm{~nm}$, the condition $\mathfrak{R e}(\bar{\varepsilon})=0$ holds, giving rise to a photonic DP; for other values of $t_{m}$, the average permittivity is shifted away from the zero, hence a BG opens.

Disorder is introduced into the nanostructure by assuming a random fluctuation of the thickness of the dielectric layers, namely, the thickness of the $n$-th layer is $t_{d}^{n}=t_{d 0}+\delta_{n}$, where $t_{d 0}$ is the average thickness (we take $t_{d 0}=256 \mathrm{~nm}$ here), and $\delta_{n}$ is a random value, uniformly distributed in the interval of $[-\delta, \delta]$, with $0<\delta<t_{d 0}$. Thus the disorder strength may be defined as $\Delta \equiv \delta / t_{d 0}$. We calculated the effective size of the emerging Anderson modes as

$$
\mathbf{W}_{\text {eff }}=\left\langle\left|\frac{\int_{-\infty}^{+\infty}|\mathbf{E}(x)|^{2}\left(x-x_{c}\right)^{2} d x}{\int_{-\infty}^{+\infty}|\mathbf{E}(x)|^{2} x d x}\right|^{\frac{1}{2}}\right\rangle,
$$

where $x_{c} \equiv \int_{-\infty}^{+\infty}|\mathbf{E}(x)|^{2} x d x / \int_{-\infty}^{+\infty}|\mathbf{E}(x)|^{2} d x$ is the center of the Anderson-localized mode, and " \langle\rangle " stands for averaging over multiple realizations of the randomness with the same strength of disorder.

Figures 4(a-d) and 4(g,h) show the effective width of the modes in the lattices of the DP and BG types, respectively, which feature a gradually increasing strength of the structural disorder. Figures $4(\mathrm{a}-\mathrm{f})$ demonstrate that the modes with the smallest and largest eigenvalues at first become localized, then the localization effect extends towards the central region of the spectrum. Nevertheless, the modes at the center of the spectrum, which correspond to the DP of the disorder-free lattice, remain delocalized despite the fact that the disorder strength attains large value [80\% in Fig. 4(d)]. As might be expected, a similar scenario is observed as well when thicknesses of the dielectric and metallic layers of the lattice unit cell are both randomized [Figs. 4(e,f)]. Spatial profiles of modes with the largest effective width are shown in inset to Figs. 4(d) and 4(f). It is worthy to note that the propagation constants of the widest mode and the corresponding DP are exactly equal. This is a clear manifestation of the extreme robustness of the ADIDP in the present setting against structural disorder, a phenomenon that can be understood by recalling that the condition for the formation of such ADIDPs is vanishing of the averaged permittivity, $\bar{\varepsilon}=0$. Indeed, if, in the disorder-
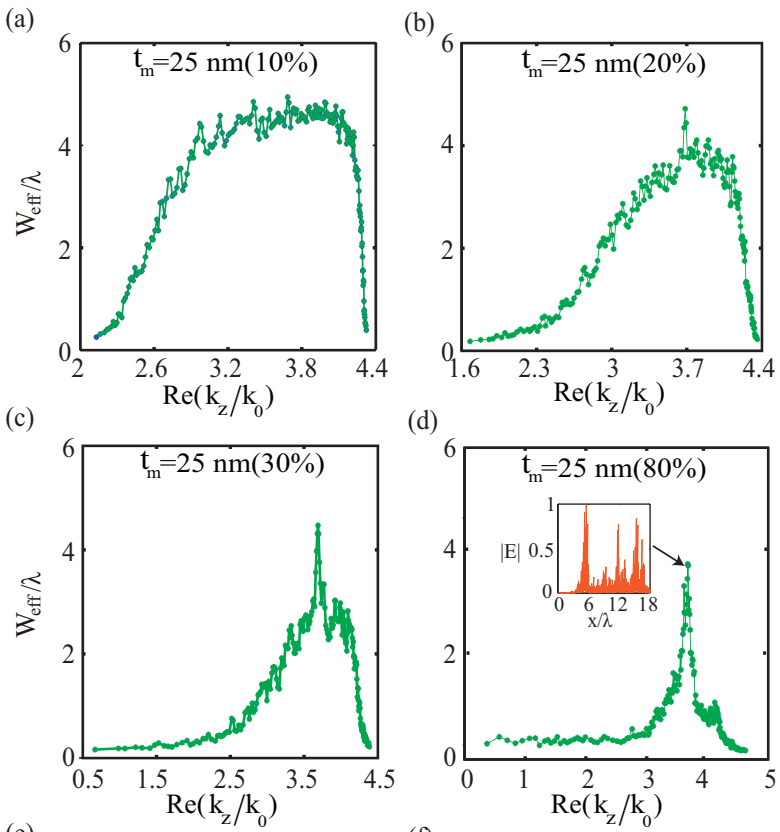

(d)

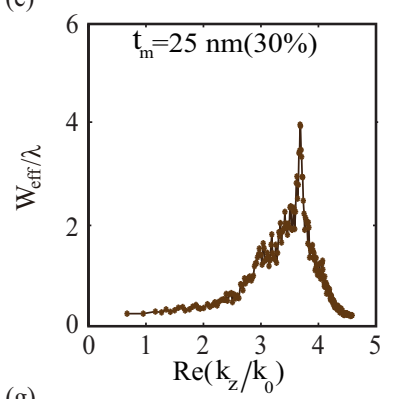

(f)
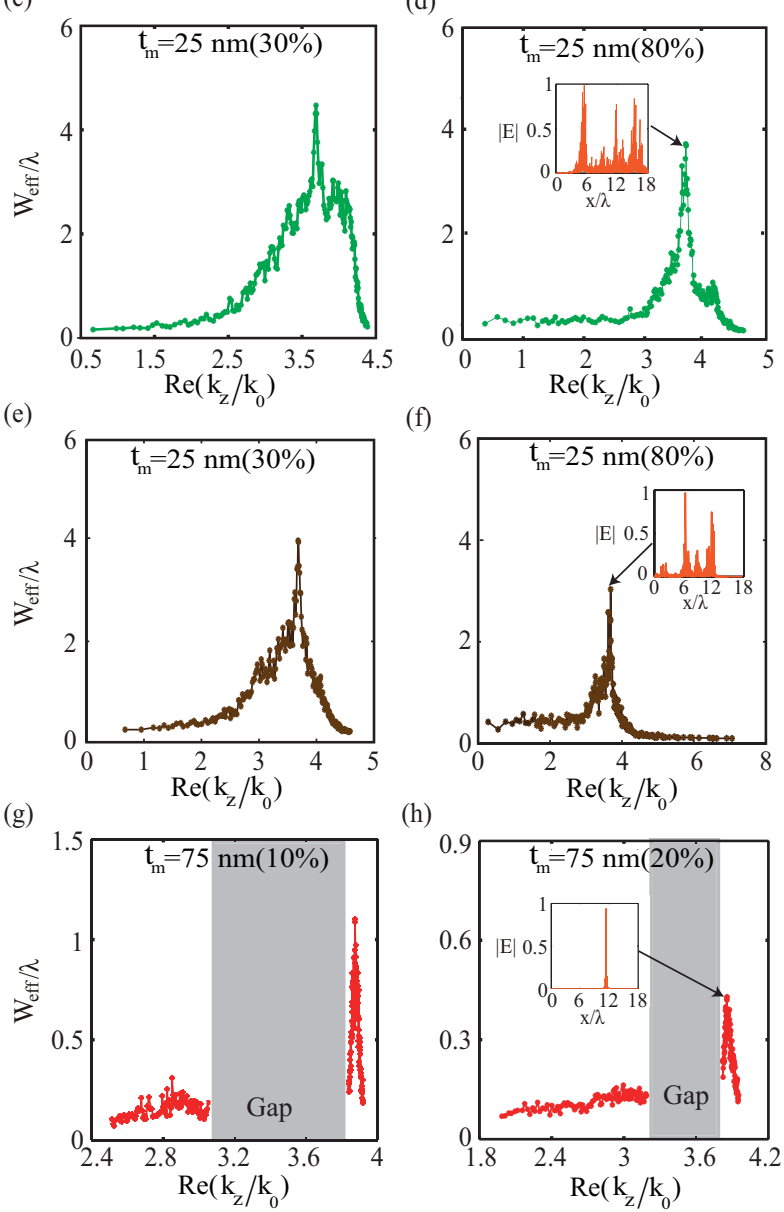

(h)
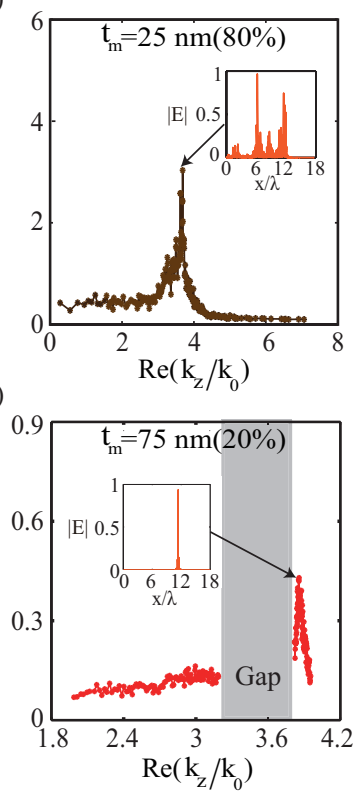

Fig. 4. The dependence of the width of the Anderson-localized modes on the disorder strength, calculated for the DP-bearing system (a)-(f), and for the BG system (g)-(h). In (e)-(f), widths of both dielectric and metallic layers are disordered. Insets in (d), (f) and (h) show profiles of the most delocalized Anderson modes. Gray regions in $(\mathrm{g})$ and $(\mathrm{h})$ indicate the bandgap of the eigenvalue spectra of the unperturbed lattices.

free lattice, one has $\bar{\varepsilon}=\left(\varepsilon_{d} t_{d 0}+\varepsilon_{m} t_{m 0}\right) /\left(t_{d 0}+t_{m 0}\right)=0$, then in the disordered system the average permittivity is

$$
\begin{aligned}
\bar{\varepsilon}= & \lim _{L \rightarrow \infty}\left\{L^{-1} \sum_{n} \varepsilon_{m}\left(t_{m 0}+\delta_{m}^{n}\right)+\sum_{n} \varepsilon_{d}\left(t_{d 0}+\delta_{d}^{n}\right)\right\} \\
= & \lim _{L \rightarrow \infty}\left\{L ^ { - 1 } \left[\sum_{n}\left(\varepsilon_{m} t_{m 0}+\varepsilon_{d} t_{d 0}\right)\right.\right. \\
& \left.\left.+\varepsilon_{m} \sum_{n} \delta_{m}^{n}+\varepsilon_{d} \sum_{n} \delta_{d}^{n}\right]\right\}=0
\end{aligned}
$$

where $L$ is the total transverse length of the disordered lattice, 

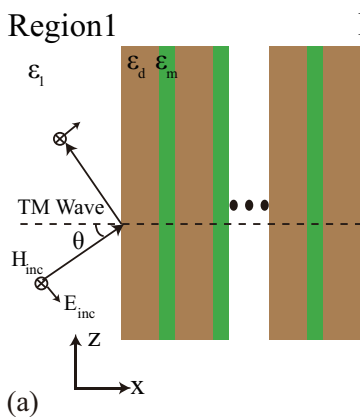

Region2

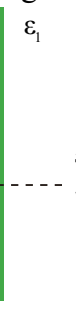

(a)

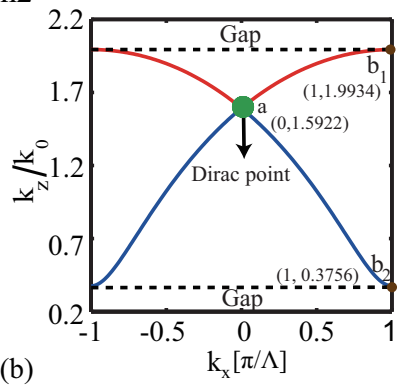

Fig. 5. (a) Schematic of the dielectric-metal layered photonic crystal (PC), stacked along the $x$ direction. A TM-polarized plane wave is launched from region 1 into the $\mathrm{PC}$ at incidence angle $\theta$ with the $+x$ direction. (b) The photonic band structure, featuring the 1D Dirac point. Parameters are $\varepsilon_{1}=$ $12.25, \varepsilon_{\mathrm{d}}=2.25, \varepsilon_{m}=-20, t_{d}=196 \mathrm{~nm}, t_{m}=22 \mathrm{~nm}, \lambda=632.8 \mathrm{~nm}$. As above, $t_{d}$ and $t_{m}$ are the thicknesses of the dielectric and metallic layers, respectively, and $\Lambda=t_{d}+t_{m}$ is the size of the lattice cell. "a" denotes the location of the Dirac point, while " $b_{1}$ " and " $b_{2}$ " are edge points of the bands.

and the use is made of the fact that the average value of the random perturbations is zero. Thus the random structural fluctuations preserve this zero-epsilon condition, hence the corresponding mode remains delocalized. By contrast, the width of the modes in the BG-bearing lattice rapidly become localized as disorder is added to the photonic lattice. This is observed in Figs. $4(\mathrm{~g}, \mathrm{~h})$, where the width of the modes corresponding to two different BG-bearing lattices are presented. In particular, one can see that even weak disorder (with $\Delta=20 \%$ ) is able to make almost all the modes localized. Even the widest mode is actually tightly localized when the disorder strength is a mere $20 \%$, as seen in the inset to Fig 4(h). It is also instructive to compare widths of the most delocalized modes in the two lattices, looking at the insets to Figs. 4(d), 4(f), and 4(h).

\section{TRANSMissivity NEAR DiRAC POINTS}

This section addresses the transmissivity of light through a finite-size plasmonic crystal. We use the same lattice model as in Section III, namely, one built of alternating layers of silicon $\left(\varepsilon_{d}=2.25\right)$ and silver $\left(\varepsilon_{m}=-20\right.$ at $\left.\lambda=632.8 \mathrm{~nm}\right)$ stacked along the $x$ axis. The lattice is bounded at each edge by a homogeneous semi-infinite dielectric medium with a permittivity $\epsilon_{1}$, labeled as Region 1 and Region 2 in Fig. 5(a). The band structure for the periodic lattice satisfying the condition of $\mathfrak{R e}(\bar{\varepsilon})=\left(t_{d}+t_{m}\right)^{-1} \mathfrak{R e}\left(\varepsilon_{d} t_{d}+\varepsilon_{m} t_{m}\right)=0$ is shown in Fig. 5(b). As expected, the DP is found at $\left(k_{x}, k_{z}\right)=\left(0,1.5922 k_{0}\right)$, with $k_{0}=2 \pi / \lambda$. Considering a plane TM-polarized wave coming from Region 1, as shown in Fig. 5(a), at incidence angle $\theta$, the transfer matrix method [32] makes it possible to calculate the transmissivity for the incident wave passing into Region 2.

\section{A. Transmission near the Dirac point in periodic lattices}

First, we consider the most interesting case when the incident plane wave has the $z$-component of the wave vector, $k_{z i}=k_{0} \sin \theta \sqrt{\varepsilon_{1}}$, that exactly corresponds to the DP. Note that the DP is located at $k_{z}=1.5922 k_{0}>k_{0}$, therefore, to match $k_{z i}$ to the DP, the permittivity in Region $1, \varepsilon_{1}$, must be larger than that of the dielectric component of the lattice, i.e., $\varepsilon_{1}>\varepsilon_{d}=2.25$. Thus, we choose silicon as the material in Region 1, with the above-mentioned large values of the permittivity, $\varepsilon_{1}=12.25$. By tuning the angle of incidence, one can readily meet the condition of $\sin \theta \sqrt{\varepsilon_{1}}=1.5922$, and then study the transmission through the lattice at the DP. Further varying the incidence angle, one can also study the transmission for $k_{z i}$ falling into allowed bands or forbidden bandgaps. The results of this analysis are summarized in Figs. 6.

Figure 6(a) shows the transmissivity at the DP which is clearly inversely proportional to the length of the lattice, $L$ (the slope of the curve at large $L$ in the log-log plot is -1 ). This finding is consistent with the conclusion of Ref. [33]. For comparison, when the incident wave number $k_{z i}$ is tuned to the bandgaps, the transmissivity is, quite naturally exponentially suppressed, see examples in Fig. 6(b) for the first finite gap, which separates the second and third band, and Fig. 6(c) for the semi-infinite gap. Finally, if the incidence angle is adjusted to put $k_{z i}$ into the allowed bands, a periodic dependence of the transmissivity on $L$ is observed in Figs. 6(c,d). This is a manifestation of the excitation of an associated Bloch-Floquet modes, that can be written as $2 f(x) \cos \left(k_{x} x\right) \exp \left(i k_{z i} z\right)$, where function $f(x)$ is the factor in the Bloch function whose period is identical to the lattice period. From this expression, one can easily see that a larger detuning of $k_{z i}$ from the DP leads to a higher oscillation frequency of the transmissivity.

\section{B. Effect of disorder on the transmissivity at the Dirac point}

As shown above, the transmissivity through the regular lattice at the DP scales as $1 / L$. A natural question is whether structural disorder destroys this feature. To address this question, we introduce structural disorder into the lattice possessing a DP as it was done in Section III. Moreover, the transmissivity is calculated as the ensemble-average over 500 disorder realizations. The results are summarized in Fig. 7.

A profound property that Figure 7(a) shows is that, the transmissivity at the DP can slightly increase with the growth of the disorder strength, as long as it remains weak enough. Physically, this is linked to the fact that Anderson modes are always excited in 1D lattices, but their localization size exceeds the finite width of the weakly disordered lattice, the tails of these modes reaching the edges of the finite lattice thus enhancing the transmission. The enhancement in the presence of disorder is even more profound for the transmission in the forbidden bandgaps, which is similar to the scenario observed before [34]. Another striking fact that Fig. 7(a) shows is that, the slopes of the transmission curve in the log-log plot are very well approximated by $T \sim 1 / L$ at large $L$ region. This means that the dependence of transmission on $1 / L$ found in the "clear" DP-bearing lattices persists when the lattices are perturbed.

Figure 7(b) further presents results when the DP-bearing lattice is perturbed by strong disorders. We see a monotonous decrease of the transmissivity with a growing disorder strength. This is expected, as when the disorder strength grows, the 
(a)
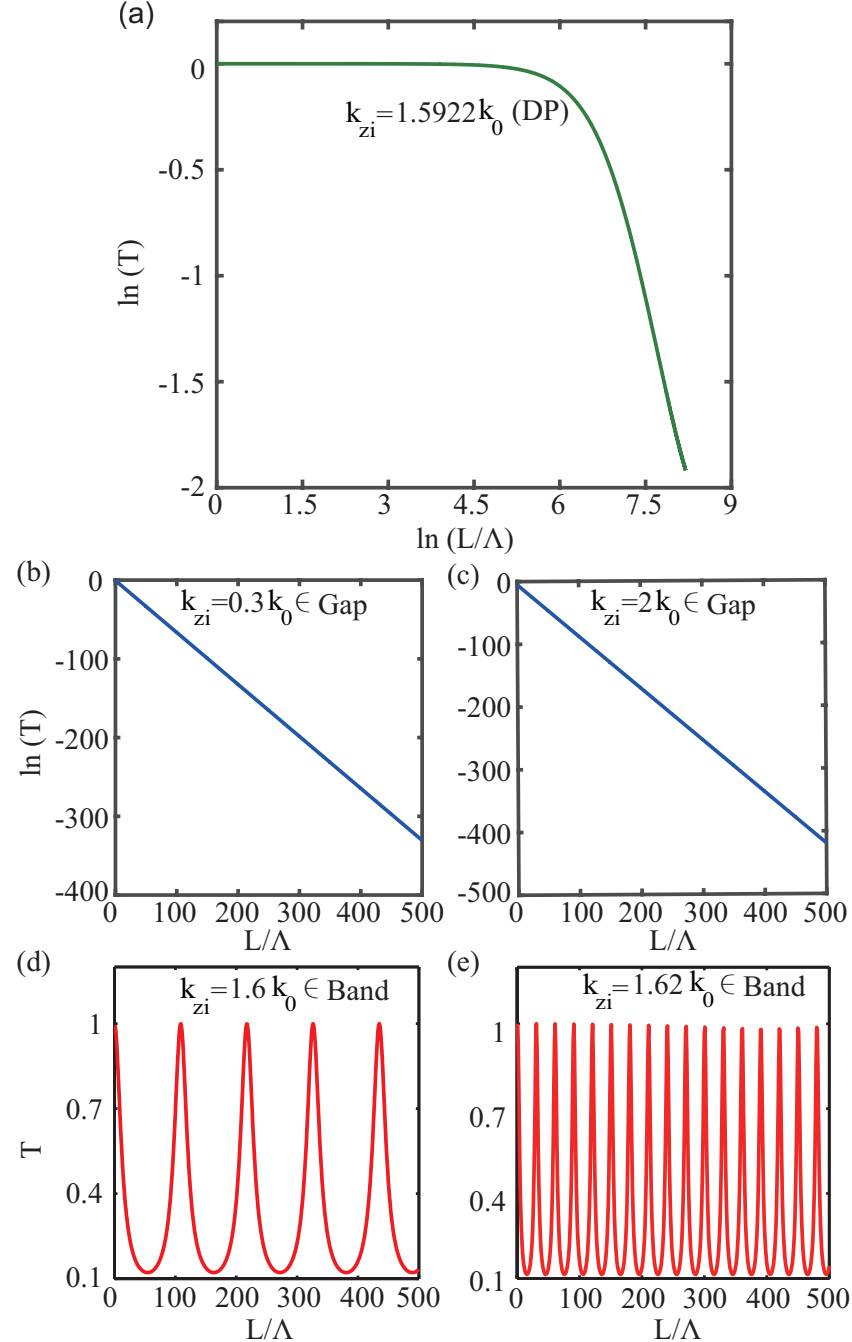

(e)

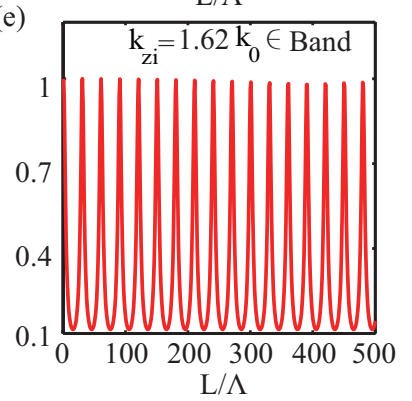

Fig. 6. The dependence of transmissivity on the propagation distance, calculated for different values of the $z$-component of the incident wave vector, $k_{z i}=k_{0} \sin \theta \sqrt{\mu_{1} \varepsilon_{1}}$. (a) $k_{z i}=1.5922 k_{0}$ at the DP. (b) $k_{z i}=0.3 k_{0}$ in the first finite forbidden gap. (c) $k_{z i}=2 k_{0}$ in the semi-infinite gap. In (d) and (e) $k_{z i}$ falls into allowed bands.

corresponding Anderson modes get localized on a scale smaller than the length of the lattice, which causes. Remarkably, though, at sufficiently large $L$, the $1 / L$ dependence of the transmissivity remains almost unaltered, although the overall transmission is strongly attenuated by strong disorder, such as that at the $80 \%$ level. The survival of the $1 / L$ scaling law of the transmissivity under the strong disorder is another important manifestation of the robustness of DPs.

\section{CONCLUSION}

We have studied the tunability and robustness of the photonic DPs (Dirac points) in plasmonic nanostructures. The tunability of the DPs was demonstrated in graphene-based photonic superlattices by tuning the graphene permittivity via the optical Kerr effect, electric gating, or chemical doping. The robustness of the DPs was demonstrated in plasmonic lattices by showing that even very high levels of disorder are unable to cause the Anderson localization of modes near the DP, and by showing that the $1 / L$ dependence of the transmissivity on size

(a)

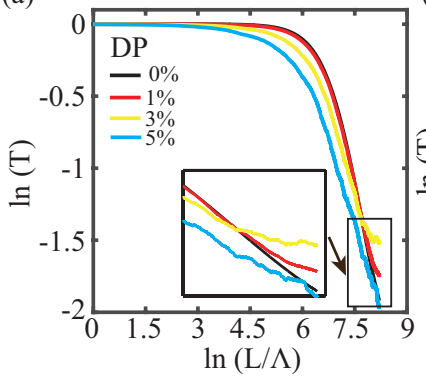

(b)

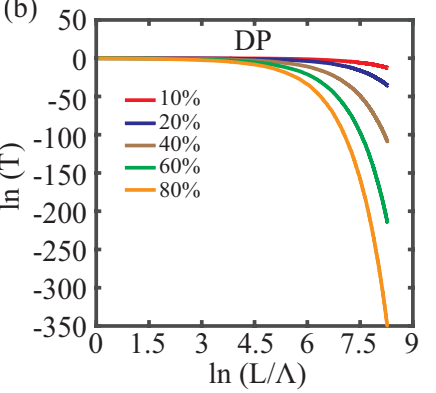

Fig. 7. The effect of the structural disorder on the transmissivity at the Dirac point. The results are obtained as the ensemble-average over 500 disorder realizations. The percentage shows the disorder strengths, low in (a), and high in (b).

$L$ of the finite lattice remains unchanged near the Dirac point under strong disorder. Our findings suggest a highly effective and robust approach for the control of photonic beams at the nanoscale, and reveal effects of the structural disorder on the functionality of certain optical nanodevices.

The approach developed in this paper may be applied to other relevant settings. One of them is the parity-time $(\mathcal{P} \mathcal{T})$ symmetry at subwavelength scales. Why the $\mathcal{P} \mathcal{T}$ symmetry is usually introduced in the paraxial approximation, assuming balance between symmetrically placed gain and loss elements [35], this property for nano-optical settings, based on the use of the full system of the Maxwell's equations, was recently put forward in Ref. [36]. In particular, the $\mathcal{P} \mathcal{T}$ symmetry may be much more robust (suffering breakup at much higher levels of the gain and loss, or remaining unbreakable) in that setting, in comparison with its paraxial counterpart. In the present context, the $\mathcal{P} \mathcal{T}$ symmetry can be introduced into the graphene-metal-dielectric superstructure by imparting equal amounts of gain and loss to alternating dielectric and metallic layers. The interplay of the DP-type dispersion and $\mathcal{P} \mathcal{T}$ symmetry in such a system will be considered elsewhere.

\section{APPENDIX A}

\section{BRIEF OUTLINE OF THE TRANSFER MATRIX METHOD}

We use the transfer matrix method (TMM) to obtain the photonic band structure and transmissivity of the structures. To this end, the electromagnetic field at two positions $x$ and $x+\Delta x$ in the same layer is related via the following transfer matrix [22], [32]:

$$
M_{j}(\Delta x, \omega)=\left(\begin{array}{cc}
\cos \left(k_{j} \triangle x\right) & \frac{i}{q_{j}} \sin \left(k_{j} \triangle x\right) \\
i q_{j} \sin \left(k_{j} \triangle x\right) & \cos \left(k_{j} \triangle x\right)
\end{array}\right),
$$

where $k_{j}=\sqrt{(\omega / c)^{2} \varepsilon_{j} \mu_{j}-k_{z}^{2}}, j=g, d, m$. Here, $g, d$, and $m$ stand for graphene, silicon, and silver, respectively. For a TM-polarized wave, $q_{j}=\left[\mu_{j} / \varepsilon_{j}\left(1-\sin ^{2} \theta / \mu_{j} \varepsilon_{j}\right)\right]^{1 / 2} . \theta$ is the angle of incidence of light(Fig. 5(a)).

\section{A. Derivation of the photonic band structure}

According to the Bloch theorem, if one considers a periodic photonic structure with period, $\Lambda$, the electric and magnetic 
components of an electromagnetic mode in layers $N$ and $N-$ 1 , with Bloch wavevector, $k_{x}$, are related to each other through,

$$
\left(\begin{array}{c}
H_{N} \\
E_{N}
\end{array}\right)=e^{i k_{x} \Lambda}\left(\begin{array}{c}
H_{N-1} \\
E_{N-1}
\end{array}\right) .
$$

On the other hand, the TMM leads to:

$$
\begin{aligned}
\left(\begin{array}{c}
H_{N} \\
E_{N}
\end{array}\right) & =M_{g} M_{d} M_{m}\left(\begin{array}{c}
H_{N-1} \\
E_{N-1}
\end{array}\right) \\
& =\left(\begin{array}{ll}
m_{11} & m_{12} \\
m_{21} & m_{22}
\end{array}\right)\left(\begin{array}{c}
H_{N-1} \\
E_{N-1}
\end{array}\right) .
\end{aligned}
$$

Combining Eqs. (6) and (7) yields:

$$
\left(\begin{array}{ll}
m_{11} & m_{12} \\
m_{21} & m_{22}
\end{array}\right)\left(\begin{array}{c}
H_{N-1} \\
E_{N-1}
\end{array}\right)=e^{i k_{x} \Lambda}\left(\begin{array}{c}
H_{N-1} \\
E_{N-1}
\end{array}\right),
$$

so that the factor $e^{i k_{x} \Lambda}$ is the eigenvalue of the transfer matrix $M=M_{g} M_{d} M_{m}$. This conclusion can be expressed as

$$
e^{i k_{x} \Lambda}=\frac{1}{2}\left(m_{11}+m_{22}\right) \pm\left[\frac{1}{4}\left(m_{11}+m_{22}\right)^{2}-1\right]^{\frac{1}{2}} .
$$

From Eq. (9) one can easily derive the dispersion relation, $k_{x}=k_{x}\left(k_{z}, \omega\right)$,

$$
k_{x}\left(k_{z}, \omega\right)=\frac{1}{\Lambda} \arccos \left[\frac{1}{2}\left(m_{11}+m_{22}\right)\right] .
$$

By substituting the elements of the matrix $M$, this relation yields,

$$
\begin{aligned}
\cos \left(k_{x} \Lambda\right)= & \cos \left(k_{g} t_{g}\right) \cos \left(k_{d} t_{d}\right) \cos \left(k_{m} t_{m}\right) \\
& -\frac{1}{2} \frac{\varepsilon_{g}^{2} k_{d}^{2}+\varepsilon_{d}^{2} k_{g}^{2}}{\varepsilon_{d} \varepsilon_{g} k_{g} k_{d}} \sin \left(k_{g} t_{g}\right) \sin \left(k_{d} t_{d}\right) \cos \left(k_{m} t_{m}\right) \\
& -\frac{1}{2} \frac{\varepsilon_{g}^{2} k_{m}^{2}+\varepsilon_{m}^{2} k_{g}^{2}}{\varepsilon_{m} \varepsilon_{g} k_{g} k_{m}} \sin \left(k_{g} t_{g}\right) \cos \left(k_{d} t_{d}\right) \sin \left(k_{m} t_{m}\right) \\
& -\frac{1}{2} \frac{\varepsilon_{m}^{2} k_{d}^{2}+\varepsilon_{d}^{2} k_{m}^{2}}{\varepsilon_{d} \varepsilon_{m} k_{d} k_{g}} \cos \left(k_{g} t_{g}\right) \sin \left(k_{d} t_{d}\right) \sin \left(k_{m} t_{m}\right) .
\end{aligned}
$$

Finally, Eq. (11) can be simplified to

$$
\begin{aligned}
& \frac{1}{\sigma} \cos \left(k_{x} \Lambda\right)=1-\frac{\varrho_{g d}^{2}+\varrho_{d g}^{2}}{2 \varrho_{g d} \varrho_{d g}} \tan \kappa_{g} \tan \kappa_{d} \\
& \quad-\frac{\varrho_{d m}^{2}+\varrho_{m d}^{2}}{2 \varrho_{d m} \varrho_{m d}} \tan \kappa_{d} \tan \kappa_{m}-\frac{\varrho_{m g}^{2}+\varrho_{g m}^{2}}{2 \varrho_{m g} \varrho_{g m}} \tan \kappa_{m} \tan \kappa_{g},
\end{aligned}
$$

where $\sigma=\cos \kappa_{g} \cos \kappa_{d} \cos \kappa_{m}$. We have also defined $\varrho_{i j}=$ $\kappa_{i} \tau_{j}, \kappa_{i}=k_{i} t_{i}$, and $\tau_{i}=\varepsilon_{i} t_{i}$, where $i, j=g, d, m$.

For a binary periodic nanostructure composed of alternating layers of metallic and dielectric materials stacked along the $x$ axis, as depicted in Fig 5(a), photonic bands of the structure are obtained by using the method just described and are given by the solutions of the following transcendental equation:

$$
\begin{aligned}
\cos \left(k_{x} \Lambda\right)= & \cos \left(k_{d} t_{d}\right) \cos \left(k_{m} t_{m}\right) \\
& -\frac{1}{2}\left(\frac{\varepsilon_{d} k_{m}}{\varepsilon_{m} k_{d}}+\frac{\varepsilon_{m} k_{d}}{\varepsilon_{d} k_{m}}\right) \sin \left(k_{d} t_{d}\right) \sin \left(k_{m} t_{m}\right) .
\end{aligned}
$$

\section{B. Derivation of the formula for lattice transmissivity}

For further convenience, we define the basic transfer matrix between two positions $x$ and $x+\Delta x$ as,

$$
M_{j}^{\prime}(\Delta x, \omega)=M_{j}(-\Delta x, \omega)
$$

where $M_{j}$ is defined in Eq.(5).

According to the transfer matrix formalism, for a PC consisting of $N$ layers the matrix associated to the lattice is given by the product of the respective transfer matrices for the individual layers. Thus, we have:

$$
\begin{aligned}
\left(\begin{array}{c}
H_{0} \\
E_{0}
\end{array}\right) & =\prod_{j=1}^{N} M_{j}^{\prime}\left(\begin{array}{c}
H_{N} \\
E_{N}
\end{array}\right) \\
& =\left(\begin{array}{ll}
m_{11}^{\prime} & m_{12}^{\prime} \\
m_{21}^{\prime} & m_{22}^{\prime}
\end{array}\right)\left(\begin{array}{c}
H_{N} \\
E_{N}
\end{array}\right) .
\end{aligned}
$$

Let $\mathcal{I}, \mathcal{R}$, and $\mathcal{T}$ denote the amplitudes (possibly complex) of the magnetic vectors of the incident, reflected, and transmitted waves, respectively. We then define the reflection coefficient as $r=\frac{\mathcal{R}}{\mathcal{I}}$, and the transmission coefficient as $t=\frac{\mathcal{T}}{\mathcal{I}}$.

The boundary conditions demand that the tangential components of the fields $E$ and $H$ are continuous across each of the two boundaries of the layered medium. In conjunction with the relation

$$
H=\sqrt{\frac{\varepsilon}{\mu}} E,
$$

one can easily show that for TM waves the following relations holds:

$$
\left(\begin{array}{c}
\mathcal{I}+\mathcal{R} \\
p_{i}(\mathcal{I}-\mathcal{R})
\end{array}\right)=\left(\begin{array}{ll}
m_{11}^{\prime} & m_{12}^{\prime} \\
m_{21}^{\prime} & m_{22}^{\prime}
\end{array}\right)\left(\begin{array}{c}
\mathcal{T} \\
p_{l} \mathcal{T}
\end{array}\right) .
$$

where $p_{i}=p_{l}=\sqrt{\frac{1}{\varepsilon_{1}}} \cos \theta$. From this relation we finally obtain a matrix equation that relates the transmission and reflection coefficients:

$$
\left(\begin{array}{c}
1+r \\
p_{i}(1-r)
\end{array}\right)=\left(\begin{array}{ll}
m_{11}^{\prime} & m_{12}^{\prime} \\
m_{21}^{\prime} & m_{22}^{\prime}
\end{array}\right)\left(\begin{array}{c}
1 \\
p_{l}
\end{array}\right) t
$$

From this equation we can derive the formula for the transmission coefficient,

$$
t=\frac{2 p_{i}}{\left(m_{11}^{\prime}+m_{12}^{\prime} p_{l}\right) p_{i}+\left(m_{21}^{\prime}+m_{22}^{\prime} p_{l}\right)} .
$$

In terms of $t$, the transmissivity of the lattice is given by:

$$
T=\frac{p_{l}}{p_{i}}|t|^{2} .
$$

\section{APPENDIX B}

\section{PHYSICAL MODELS FOR THE ELECTRIC PERMITTIVITY OF} METAL AND GRAPHENE

For the metallic layers of our photonic superlattice we use the Drude model for the complex, frequency-dependent electric permittivity of the metal:

$$
\varepsilon_{m}=1-\frac{\omega_{p}^{2}}{\omega(\omega+i \nu)},
$$

where $\omega_{p}$ and $\nu$ are the plasma and damping frequencies, respectively. In the paper, the metallic layers are assumed 
to be made of silver, with $\omega_{p}=13.7 \times 10^{15} \mathrm{rad} \mathrm{s}^{-1}$ and $\nu=2.7 \times 10^{13} \mathrm{rad} \mathrm{s}^{-1}$ [37]. For graphene, we use the Kubo's formula to model the surface conductivity, $\sigma_{s}$, including the contributions of both the interband and intraband transitions [38],

$$
\begin{aligned}
& \sigma_{s}\left(\omega, \mu_{c}, \tau, T\right)=-\frac{i e^{2}\left(\omega+i \tau^{-1}\right)}{\pi \hbar^{2}}\left\{\int_{-\infty}^{+\infty} \frac{\partial f(\epsilon)}{\partial \epsilon} \frac{|\epsilon| d \epsilon}{\left(\omega+i \tau^{-1}\right)^{2}}\right. \\
& \left.-\int_{0}^{+\infty}\left[\frac{\partial f(-\epsilon)}{\partial \epsilon}-\frac{\partial f(\epsilon)}{\partial \epsilon}\right] \frac{d \epsilon}{\left(\omega+i \tau^{-1}\right)^{2}-4(\epsilon / \hbar)^{2}}\right\} .
\end{aligned}
$$

Here, $f(\epsilon)=1 /\left\{1+\exp \left[\left(\epsilon-\mu_{c}\right) /\left(k_{B} T\right)\right]\right\}$ is the FermiDirac distribution, with $k_{B}$ being the Boltzmann constant, $\epsilon$ the energy, $\mu_{c}$ the chemical potential, $T$ the temperature, $e$ the electron charge, $\hbar$ the reduced Planck's constant, and $\tau$ the momentum relaxation time (inverse of the electronphonon scattering rate). A carrier density as large as $n \approx$ $2 \times 10^{14} \mathrm{~cm}^{-2}$ has been achieved experimentally with an ionic liquid gating [39], which corresponds to a Fermi level $\mu_{c}=1.65 \mathrm{eV}\left(\mu_{c}=\hbar V_{\mathrm{F}} \sqrt{\pi n}\right.$, see [40]). We also note that $n \approx 4 \times 10^{14} \mathrm{~cm}^{-2}$ has been realized with an electrolytic gate [41], which means $\mu_{c}=2.33 \mathrm{eV}$. For such gating schemes, the typical scattering time is of the order of $100 \mathrm{fs}$ [42]. We used in Eq. (21) the experimentally measured value, $\tau=90 \mathrm{fs}$ [42].

The first term in Eq. (21) corresponds to intraband electronphoton scattering processes, and can be evaluated to

$$
\sigma_{\text {intra }}=i \frac{e^{2} k_{B} T}{\pi \hbar^{2}\left(\omega+i \tau^{-1}\right)}\left[\frac{\mu_{\mathrm{c}}}{k_{B} T}+2 \ln \left(e^{-\frac{\mu_{c}}{k_{\mathrm{B}} T}}+1\right)\right],
$$

whereas the second term corresponds to direct interband electron transitions and, for $\hbar \omega,\left|\mu_{c}\right| \gg k_{B} T$, it can be approximated to:

$$
\sigma_{\text {inter }}=i \frac{e^{2}}{4 \pi \hbar} \ln \left[\frac{2\left|\mu_{c}\right|-\hbar\left(\omega+i \tau^{-1}\right)}{2\left|\mu_{c}\right|+\hbar\left(\omega+i \tau^{-1}\right)}\right] .
$$

\section{APPENDIX C \\ THE LINEAR AND NONLINEAR PERMITTIVITY OF GRAPHENE}

For the sake of convenience in numerical simulations, graphene layers have been approximated by finite-thickness layers with the effective dielectric constant given by [43], [44]:

$$
\begin{aligned}
\varepsilon_{g, t o t} & =1+\frac{i \sigma_{g, \text { tot }} \eta_{0}}{k_{0} t_{g}}=1+\frac{i \eta_{0}}{k_{0} t_{g}}\left(\sigma_{s}+\sigma_{3}\left|\mathrm{E}_{\|}\right|^{2}\right) \\
& =\varepsilon_{g}+\chi_{g}^{(3)}\left|\mathrm{E}_{\|}\right|^{2},
\end{aligned}
$$

where $\sigma_{g}$ is the total conductivity of graphene layer, including both the linear and nonlinear contributions, namely, $\sigma_{g}=$ $\sigma_{s}+\sigma_{3}\left|\mathrm{E}_{\|}\right|^{2} . \eta_{0}$ is the vacuum impedance $\left(\eta_{0}=\sqrt{\mu_{0} / \epsilon_{0}} \approx\right.$ $377 \Omega), t_{g}$ is the thickness of graphene $\left(t_{g}=0.5 \mathrm{~nm}\right.$ used in our simulations), $\mathrm{E}_{\|}$is the in-plane component of electric field, and $k_{0}=2 \pi / \lambda$ is the wavenumber in vacuum of light with wavelength, $\lambda$. The nonlinear contribution to the surface conductivity of the graphene is given by, $\sigma_{3}\left|\mathrm{E}_{\|}\right|^{2}$, where $\sigma_{3}$ is the nonlinear conductivity coefficient, expressed as [30], [45]:

$$
\sigma_{3}=-i \frac{3}{32} \frac{e^{4} v_{F}^{2}}{\pi \hbar^{2} \mu_{c} \omega^{3}}
$$

In this equation, $v_{F} \approx \mathrm{c} / 300$ is the Fermi velocity. Equivalently, one can define a third-order effective nonlinear susceptibility, $\chi_{g}^{(3)}=\frac{i \sigma_{3} \eta_{0}}{k_{0} t_{g}}$. If we assume $\mu_{c}=1.5 \mathrm{eV}$ and $\lambda=4.2 \mu \mathrm{m}$, as we used in our calculations, one finds $\chi_{g}^{(3)}=4.09 \times 10^{-17} \mathrm{~m}^{2} \mathrm{~V}^{-2}$.

\section{REFERENCES}

[1] S. Nam, A. Taylor, and A. Efimov, "Diabolical point and conical-like diffraction in periodic plasmonic nanostructures," Opt. Express, vol. 18, no. 10, pp. 10120-10126, 2010.

[2] L. Sun, G. Gao, and X. Yang, "Giant optical nonlocality near the dirac point in metal-dielectric multilayer metamaterials," Opt. Express, vol. 21, no. 18, pp. 21542-21555, 2013.

[3] H. Deng, X. Chen, B. A. Malomed, N. C. Panoiu, and F. Ye, "Transverse Anderson localization of light near Dirac points of photonic nanostructures," Sci. Rep., vol. 5, art. no. 15585, 2015.

[4] A. K. Geim and K. S. Novoselov, "The rise of graphene," Nat. Mater. vol. 6, no. 3, pp. 183-191, 2007.

[5] K. Novoselov, A. K. Geim, S. Morozov, D. Jiang, M. Katsnelson, I. Grigorieva, S. Dubonos, and A. Firsov, "Two-dimensional gas of massless Dirac fermions in graphene," Nature, vol. 438, no. 7065, pp. 197-200, 2005.

[6] M. C. Rechtsman, J. M. Zeuner, Y. Plotnik, Y. Lumer, D. Podolsky, F. Dreisow, S. Nolte, M. Segev, and A. Szameit, "Photonic Floquet topological insulators," Nature, vol. 496, no. 7444, pp. 196-200, 2013.

[7] J. Topolancik, B. Ilic, and F. Vollmer, "Experimental observation of strong photon localization in disordered photonic crystal waveguides," Phys. Rev. Lett., vol. 99, no. 25, p. 253901, 2007.

[8] F. Bonaccorso, Z. Sun, T. Hasan, and A. Ferrari, "Graphene photonics and optoelectronics," Nature photonics, vol. 4, no. 9, pp. 611-622, 2010.

[9] J. Chen, M. Badioli, P. Alonso-González, S. Thongrattanasiri, F. Huth, J. Osmond, M. Spasenović, A. Centeno, A. Pesquera, P. Godignon et al., "Optical nano-imaging of gate-tunable graphene plasmons," Nature, vol. 487, no. 7405, pp. 77-81, 2012.

[10] F. H. Koppens, D. E. Chang, and F. J. Garcia de Abajo, "Graphene plasmonics: a platform for strong light-matter interactions," Nano Lett., vol. 11, no. 8, pp. 3370-3377, 2011.

[11] H. Yan, X. Li, B. Chandra, G. Tulevski, Y. Wu, M. Freitag, W. Zhu, P. Avouris, and F. Xia, "Tunable infrared plasmonic devices using graphene/insulator stacks," Nat. Nanotechnol., vol. 7, no. 5, pp. 330334, 2012.

[12] Y. Yao, M. A. Kats, P. Genevet, N. Yu, Y. Song, J. Kong, and F. Capasso, "Broad electrical tuning of graphene-loaded plasmonic antennas," Nano Lett., vol. 13, no. 3, pp. 1257-1264, 2013.

[13] M. A. Othman, C. Guclu, and F. Capolino, "Graphene-dielectric composite metamaterials: evolution from elliptic to hyperbolic wavevector dispersion and the transverse epsilon-near-zero condition," J. Nanophotonics, vol. 7, no. 1, pp. 073 089-073 089, 2013.

[14] L. Yang, C. Pei, A. Shen, C. Zhao, Y. Li, T. Dai, H. Yu, Y. Li, X. Jiang, and J. Yang, "Ultracompact plasmonic switch based on graphene-silica metamaterial," Applied Physics Letters, vol. 104, no. 21, p. 211104, 2014.

[15] I. S. Nefedov, C. A. Valaginnopoulos, and L. A. Melnikov, "Perfect absorption in graphene multilayers," Journal of Optics, vol. 15, no. 11, p. 114003, 2013.

[16] O. Peleg, G. Bartal, B. Freedman, O. Manela, M. Segev, and D. N. Christodoulides, "Conical diffraction and gap solitons in honeycomb photonic lattices," Phys. Rev. Lett., vol. 98, no. 10, p. 103901, 2007.

[17] X. Huang, Y. Lai, Z. H. Hang, H. Zheng, and C. Chan, "Dirac cones induced by accidental degeneracy in photonic crystals and zerorefractive-index materials," Nat. Mater., vol. 10, no. 8, pp. 582-586, 2011.

[18] J. M. Zeuner, M. C. Rechtsman, S. Nolte, and A. Szameit, "Edge states in disordered photonic graphene," Opt. Lett., vol. 39, no. 3, pp. 602-605, 2014. 
[19] J. Tworzydło, B. Trauzettel, M. Titov, A. Rycerz, and C. W. Beenakker, "Sub-poissonian shot noise in graphene," Phys. Rev. Lett., vol. 96, no. 24, p. 246802, 2006.

[20] M. Katsnelson, "Zitterbewegung, chirality, and minimal conductivity in graphene," The European Physical Journal B-Condensed Matter and Complex Systems, vol. 51, no. 2, pp. 157-160, 2006.

[21] H. Deng, F. Ye, B. A. Malomed, X. Chen, and N. C. Panoiu, "Optically and electrically tunable Dirac points and Zitterbewegung in graphenebased photonic superlattices," Phys. Rev. B, vol. 91, no. 20, p. 201402, 2015.

[22] P. Yeh, A. Yariv, and C.-S. Hong, "Electromagnetic propagation in periodic stratified media. i. general theory," JOSA, vol. 67, no. 4, pp. 423-438, 1977.

[23] R. Hedge and H. Winful, "Zero- $\bar{n}$ gap solitons," Opt. Lett., vol. 30, no. 14 , pp. $1852-1854,2005$

[24] R. Ziolkowski,'Propagation in and scattering from a matched metamaterial having a zero index of refraction," Phys. Rev. E, vol. 70, no. 4, pp. 046608, 2004

[25] B. Schwartz and R. Piestun, "Total external reflection from metamaterials with ultralow refractive index," J. Opt. Soc. Am B , vol. 20, no. 12, pp. 2448-2453, 2003

[26] E. Vesseur, T. Coenen, H. Caglayan, N. Engheta, and A. Polman, "Experimental verification of $n=0$ structures for visible light," Phys. Rev. Lett., vol. 110, no. 1, pp.013902, 2013.

[27] N. C. Panoiu, R. M. Osgood Jr, S. Zhang, S. R. Brueck, "Zero- $\bar{n}$ bandgap in photonic crystal superlattices," J. Opt. Soc. Am. B, vol. 23, no. 3, pp. 506-513, 2006.

[28] S. Kocaman, R. Chatterjee, N. C. Panoiu, J. McMillan, M. Yu, R. M. Osgood Jr, D. Kwong, and C. Wong, "Observation of zeroth-order band gaps in negative-refraction photonic crystal superlattices at near-infrared frequencies," Phys. Rev. Lett., vol. 102, no. 20, p. 203905, 2009.

[29] S. Kocaman, M. Aras, P. Hsieh, J. McMillan, C. Biris, N. C. Panoiu, M. Yu, D. Kwong, A. Stein, and C. Wong, "Zero phase delay in negativerefractive-index photonic crystal superlattices," Nature Photonics, vol. 5, no. 8, pp. 499-505, 2011

[30] E. Hendry, P. J. Hale, J. Moger, A. Savchenko, and S. Mikhailov, "Coherent nonlinear optical response of graphene," Phys. Rev. Lett., vol. 105, no. 9, p. 097401, 2010.

[31] P. B. Johnson and R.-W. Christy, "Optical constants of the noble metals," Phys. Rev. B, vol. 6, no. 12, p. 4370, 1972.

[32] M. Born and E. Wolf, Principles of optics: electromagnetic theory of propagation, interference and diffraction of light. Cambridge university press, 1999.

[33] R. Sepkhanov, Y. Bazaliy, and C. Beenakker, "Extremal transmission at the Dirac point of a photonic band structure," Phys. Rev. A, vol. 75, no. 6, pp. 063813, 2007.

[34] V. Freilikher, B. Liansky, I. Yurkevich, A. Maradudin, and A. McGurn, "Enhanced transmission due to disorder," Phys. Rev. E, vol. 51, no. 6, p. 6301, 1995

[35] K. G. Makris, R. El-Ganainy, D. N. Christodoulides, and Z. H. Musslimani, " $\mathcal{P} \mathcal{T}$-symmetric periodic optical potentials", International Journal of Theoretical Physics, vol. 50, p. 1019, 2011.

[36] C. Huang, F. Ye, Y. V. Kartashov, B. A. Malomed, and X. Chen, "PT symmetry in optics beyond the paraxial approximation", Opt. Lett., vol. 30, p. 5443, 2014.

[37] M. A. Ordal, R. J. Bell, R. Alexander, L. Long, and M. Querry, "Optical properties of fourteen metals in the infrared and far infrared: $\mathrm{Al}, \mathrm{co}, \mathrm{cu}$, au, fe, pb, mo, ni, pd, pt, ag, ti, v, and w." Appl. opt., vol. 24, no. 24, pp. 4493-4499, 1985.

[38] L. Falkovsky and S. Pershoguba, "Optical far-infrared properties of a graphene monolayer and multilayer," Phys. Rev. B, vol. 76, no. 15, p. 153410, 2007.

[39] J. Ye, M. F. Craciun, M. Koshino, S. Russo, S. Inoue, H. Yuan, H. Shimotani, A. F. Morpurgo, and Y. Iwasa, "Accessing the transport properties of graphene and its multilayers at high carrier density," Proc. Nati. Acad. Sci., vol. 108, no. 32, pp. 13 002-13 006, 2011.

[40] Y. Zhang, Y.-W. Tan, H. L. Stormer, and P. Kim, "Experimental observation of the quantum hall effect and berry's phase in graphene," Nature, vol. 438, no. 7065, pp. 201-204, 2005.

[41] D. K. Efetov and P. Kim, "Controlling electron-phonon interactions in graphene at ultrahigh carrier densities," Phys. Rev. lett., vol. 105, no. 25, p. 256805, 2010.

[42] P. Tassin, T. Koschny, and C. M. Soukoulis," Graphene for terahertz applications," Science, vol. 341, p. 620-621, 2013.

[43] A. Vakil and N. Engheta, "Transformation optics using graphene," Science, vol. 332, no. 6035, pp. 1291-1294, 2011.
[44] E. Forati, G. W. Hanson, A. B. Yakovlev, and A. Alù, "Planar hyperlens based on a modulated graphene monolayer," Phys. Rev. B, vol. 89, no. 8, p. 081410, 2014.

[45] S. Mikhailov, "Non-linear electromagnetic response of graphene," $E P L$, vol. 79 , no. 2, p. $27002,2007$.

Hanying Deng received the B.Sc. degree from Gannan Normal University, Ganzhou, China, in 2012. She is currently working toward the Ph.D. degree under the supervision of F. Ye at Shanghai Jiao Tong University, Shanghai, China. Her research interests include nano-photonics, nonlinear Optics, numerical analysis and simulation of electromagnetic problems.

Xianfeng Chen received the Ph.D. degree in optics from Shanghai Jiao Tong University, Shanghai, China, in 1997. He is now a distinguished professor in Department of Physics and Astronomy, Shanghai Jiao Tong University. He is the recipient of China National Funds for Distinguished Young Scientists in 2011 and he won the C.N. Yang Award from AAPPS for the contribution to quasi-phase-matching nonlinear optics in 2010. During the past decade, his researchers have focused on nonlinear optics, nano photonics, laser physics and technology, etc. He has published more than 200 refereed journal articles.

Boris A. Malomed received his $\mathrm{PhD}$ degree from the Moscow PhysicoTechnical Institute (Russia) in 1981, and a Doctor of Science degree (habilitation) from the Institute for Theoretical Physics of the Academy of Sciences of Ukraine (Kiev) in 1989. He has been working at the Tel Aviv University (currently, as a professor with chair "Optical Solitons") since 1991. Since 2011, he has also been collaborating, as a consultant, with the Institute of Photonic Sciences (ICFO, Barcelona, Spain). He has served as a Divisional Associate Editor of Physical Review Letters (responsible for the area of "laser physics") in 2009-2015. His research interests are in the fields of nonlinear optics, Bose-Einstein condensates and matter waves, pattern formation in nonlinear dissipative media, dynamics of nonlinear lattices, etc. His current h-index is 63 (as per Web of Science and Scopus) and 76 (as per Google Scholar).

Nicolae C. Panoiu received the B.Sc. and M.S. degrees in physics from the University of Bucharest, Bucharest, Romania, in 1990 and 1992, respectively, and the Ph.D. degree from New York University, New York, in 2001. After graduating from NYU, he was a Postdoctoral Fellow in the Department of Applied Physics and Applied Mathematics, Columbia University, New York. $\mathrm{He}$ is currently Reader in Nanophotonics in the Department of Electronic and Electrical Engineering, University College London. His research interests include silicon photonics, optical properties of photonic nanostructures and metamaterials, and computational modeling of electromagnetic structures. Dr. Panoiu is a member of the OSA and IEEE.

Fangwei Ye received the Ph.D degree in optics from University of Science and Technology of China, Hefei, China, in 2005. From 2005 to 2008 he was a Postdoctoral Research Fellow at ICFO (The Institute of Photonic Sciences, Barcelona, Spain). From 2008 to 2010, he was a Senior Research Fellow in the Physics Department, Hong Kong Baptist University, Hong Kong. In 2010, he joined in Shanghai Jiao Tong University(SJTU). Currently he is an associate professor in the Department of Physics and Astronomy(SJTU). His research interests focus on nanophotonics and nonlinear optics. 